\title{
Inference of the Neutron Star Equation of State from Cosmological Distances
}

\author{
Carl-Johan Haster $\odot,{ }^{1,2}$ Katerina Chatziioannou, ${ }^{3}$ Andreas Bauswein $\odot,{ }^{4,5}$ and James Alexander Clark ${ }^{6}$ \\ ${ }^{1}$ LIGO Laboratory, Massachusetts Institute of Technology, 185 Albany Street, Cambridge, Massachusetts 02139, USA \\ ${ }^{2}$ Department of Physics and Kavli Institute for Astrophysics and Space Research, Massachusetts Institute of Technology, \\ 77 Massachusetts Ave, Cambridge, Massachusetts 02139, USA \\ ${ }^{3}$ Center for Computational Astrophysics, Flatiron Institute, 162 5th Avenue, New York, New York 10010, USA \\ ${ }^{4}$ GSI Helmholtzzentrum für Schwerionenforschung, Planckstraße 1, 64291 Darmstadt, Germany \\ ${ }^{5}$ Helmholtz Research Academy Hesse for FAIR (HFHF), GSI Helmholtz Center for Heavy Ion Research, \\ Campus Darmstadt, 64291 Darmstadt, Germany \\ ${ }^{6}$ Center for Relativistic Astrophysics and School of Physics, Georgia Institute of Technology, Atlanta, Georgia 30332, USA
}

(Received 27 April 2020; revised 23 August 2020; accepted 2 November 2020; published 21 December 2020)

Finite-size effects on the gravitational wave signal from a neutron star merger typically manifest at high frequencies where detector sensitivity decreases. Proposed sensitivity improvements can give us access both to stronger signals and to a myriad of weak signals from cosmological distances. The latter will outnumber the former and the relevant part of the signal will be redshifted towards the detector's most sensitive band. We study the redshift dependence of information about neutron star matter and find that single-scale properties, such as the star radius or the postmerger frequency, are better measured from the distant weak sources from $z \sim 1$.

DOI: 10.1103/PhysRevLett.125.261101

Introduction.-The gravitational wave (GW) signal emitted during the coalescence of two neutron stars (NSs) carries the imprint of the equation of state (EoS) of cold, supranuclear matter [1,2]. Extracting EoS attributes is a key science goal not only of GW astronomy but also of diverse probes such as nuclear experiment and theory, and radio and x-ray observations of pulsars [3-7]. To date, GWs from two binary neutron star (BNS) coalescences have been detected [8-11] and combined with other constraints to study the EoS, e.g., [10,12-16]. Projections from simulated observations suggest GWs will dominate the astrophysical EoS constraints in the next $\sim 5$ years [16].

Ground-based GW detectors observe a BNS coalescence for minutes, starting at $\sim 10-20 \mathrm{~Hz}$ (determined by the detector sensitivity) with increasing frequency towards merger. The final part of the signal nominally falls within the detector bandwidth, but depending on the system mass and EoS the decreased detector sensitivity might make its accurate extraction challenging in the near future. The signal below $\mathcal{O}(100) \mathrm{Hz}$ is not expected to inform NS EoS constraints as the inspiraling bodies are sufficiently separated such that matter effects are subdominant (excluding potential resonances [17,18], instabilities [19,20], etc.); scaling as $(R / r)^{5}$, where $R$ is the NS radius and $r$ the binary separation [21].

Once the NSs are sufficiently close and the signal reaches a few hundred $\mathrm{Hz}$, mutual tidal interactions induce quadrupole moments on each star. The additional quadrupole moment (besides the orbital one) affects both the binding energy and the rate of energy extraction, thus decreasing the coalescence's lifetime. This speed-up depends on the NSs size (less compact NSs being more deformable) and can be measured from the phase evolution of the signal, constraining the NSs deformability. Depending on the NS mass and radius, the part of the signal influenced by tidal interactions corresponds to frequencies $\gtrsim 400 \mathrm{~Hz}$ in the source frame of the BNS. After the inevitable collision, and unless it promptly forms a black hole $(\mathrm{BH})$, the merger remnant emits a signal dominated by a single frequency component, usually at $1500-4000 \mathrm{~Hz}$ [22-36]. This mode has been linked to $f$ modes of the remnant star [37,38]; it depends on the remnant's mean density, so it can offer complementary information about the EoS stiffness at different density and temperature conditions than the tidal deformability of the inspiraling stars. For less massive NSs or for stiffer EoSs the dominant frequency is lower and the signal is easier to extract from noise [28]. Most postmerger frequency relations in the literature relate the oscillation frequency of the rotating massive remnant to stellar properties of (fiducial) nonrotating NSs with lower mass, chosen to effectively characterize the EoS at the remnant's density that is higher than in the progenitor stars. In this sense these relations are purely empirical, as one cannot unambiguously define radius and mass of the remnant. Figure 13 in [28], however, suggests a relation between the remnant's mean density and oscillation frequency similar to that of isolated stars [39].

The two observed BNS signals already constrain tidal interactions between coalescing NSs $[9,10,40,41]$, but the postmerger signal was lost in the detector noise $[9,42]$. 
Planned improvements of GW detectors [43-46] will not only allow for better tidal measurements but might also reveal the elusive postmerger signal [47-51]. Further ahead, next generation detectors [52-56] predict an $\mathcal{O}(10)$ sensitivity increase, potentially detecting and individually resolving a significant fraction of all BNS coalescences even at cosmological distances [57], occurring every $\mathcal{O}(10 \mathrm{~s})$ [58].

The cosmological reach of next generation detectors results in observations with non-negligible redshifting compared to the emitted signal. Cosmological redshifting is familiar in GW astronomy as even current-sensitivity binary black hole (BBH) signals are appreciably redshifted [59]. This can be a blessing and a curse for BBHs as redshifting simultaneously amplifies the signal (since masses appear larger) and shifts it to lower frequencies, potentially outside the detectors' sensitive band. Observed BNS signals will also appear more massive and lower in frequency, resulting in some early inspiral signal being lost to low-frequency noise. However, for the orientation-averaged BNS reach of next generation detectors, $z \sim 2-3$ [60], this early signal is generally not informative about finite-size effects. The late inspiral and postmerger signals are typically emitted above $400 \mathrm{~Hz}$, so even a serendipitous detection at $z=10$ would redshift the signal to $\gtrsim 35 \mathrm{~Hz}$, safely above the noise dominated frequencies. Figure 1 shows the observed premerger (top) and postmerger (bottom) BNS signal at different
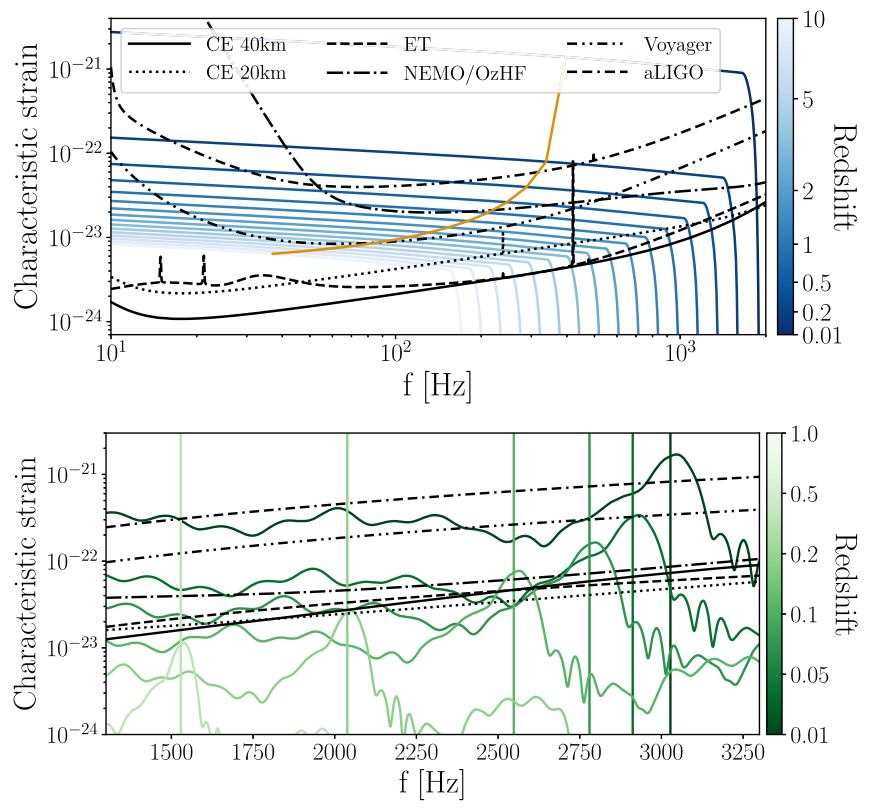

FIG. 1. Charachteristic strain [61] of a source-frame $1.362+$ $1.362 \mathrm{M}_{\odot}$ BNS signal at different redshifts (shaded blue or green) and planned or proposed detectors (black lines). Top: late-inspiral signal assuming the H4 EoS [62]. The orange line represents $400 \mathrm{~Hz}$ in the source frame; the tidally corrected signal is always observed with $f>10 \mathrm{~Hz}$. Bottom: postmerger signal assuming EoS7 from [50]. Vertical lines mark the dominant postmerger frequency. redshifts compared to various detector noise spectra. Both signals move to lower frequencies with increasing redshift, but always above $10 \mathrm{~Hz}$.

Besides redshifting, distant sources are also more numerous. The number of binaries per time, per redshift slice in the observer frame is

$$
R(z)=\frac{d N}{d T d z}=\frac{R_{s}(z)}{1+z} \frac{d V_{c}}{d z}
$$

where $d V_{c} / d z$ is the differential comoving volume, $R_{s}(z)$ is the rate per comoving volume in the source frame, and the $1+z$ term converts from the source frame to the observer frame. We assume $R_{s}(z)$ has the same shape as the star formation rate from [63], peaking at $z \sim 2$ and corresponding to a zero time delay between binary formation and merger. A nonzero time delay would smoothen the redshift-dependent rate, making it less peaked than the star formation rate. We assume a local BNS merger rate as $R_{s}(z=0)=500 \mathrm{Gpc}^{-3} \mathrm{yr}^{-1}$, consistent with current observations [11].

We study whether the fewer, loud, close-by signals or the numerous, weak, distant, redshifted signals offer the most information about the NS EoS. Expectedly, the answer depends on the detector cosmological reach. Next-generation detectors can extract a single EoS scale, such as the NS radius at a fiducial mass or the frequency of the postmerger oscillations, better with the multitude of far-away sources, thanks to appreciable nonlocal information. Conversely, detailed features of the EoS such as the functional dependence of tidal properties and radius on the mass require simultaneous extraction of multiple parameters with constraints dominated by the few loud sources from the local Universe.

Premerger signal.-During the late inspiral phase of a BNS coalescence, matter effects are encoded in the parameter $\Lambda$ quantifying the tidal deformation of a NS under an external field $[21,64,65]$. We estimate the expected information gained from measuring the tidally corrected signal and $\Lambda$ through the Fisher information matrix $F$ [66]. The accumulated information can be approximated by $\operatorname{det} F$, the determinant of $F$ which scales with the observed signal-tonoise ratio (SNR) as det $F \sim \mathrm{SNR}^{2 D}$, where $D$ is the number of relevant parameters [67]. For $D=1$ this reduces to the well-known $\mathrm{SNR}^{2}$ dependence on the measurement covariance.

$\Lambda$ depends on the NS mass $m$, making $D$ large when characterizing a generic $\Lambda(m)$ curve. In practice, most EoSs can be approximated with a few parameters only. For hadronic EoSs, only a single parameter is expected to be measurable with current detectors, for example $\Lambda\left(m=1.4 \mathrm{M}_{\odot}\right)$ [68]. Such a measurement would only provide a single EoS scaling, such as the radius at a fiducial mass. EoS-insensitive relations also suggest that hadronic EoSs can be suitably described by a single 
parameter [40,69-72]. Instead, exploring features of the EoS, such as the mass dependence of the tidal parameter or the presence of a phase transition [73-77], relies on EoS parametrizations. Common models employ $\sim 4$ or more parameters [78-84] with additional parameters for eventual phase transitions [84-86]. With this in mind we present results for $D=\{1,2\}$. The proportionality constant in $\operatorname{det} F \sim \mathrm{SNR}^{2 D}$ nominally can be parameter dependent, which could make it redshift dependent due to selection effects. However, the measurement accuracy of $\Lambda$ does not depend strongly on its true value [87], so we assume that the constant is redshift independent and does not affect the following results.

We simulate signals from one year's worth of nonspinning BNS coalescences with the redshift distribution of Eq. (1). We use WFF1 [88], SLY2 [89,90], and H4 [62] as fiducial EoSs with varying stiffness, but consistent with GW170817 [10,91]. We assume two source-frame mass distributions: (i) masses drawn uniformly in $\left[1, M_{\max }\right] \mathrm{M}_{\odot}$, where $M_{\max }$ is the assumed EoS maximum mass, and (ii) the primary mass drawn from a bimodal distribution [92] with a mass ratio $q \equiv m_{2} / m_{1}<1$ distribution $\sim q^{3}$ [93]. We distribute systems uniformly across the sky and binary orientations and retain systems with single-detector SNR above 8, evaluated from $10 \mathrm{~Hz}$ with the waveform model IMRPHENOMPV2_NRTIDAL [94] and the proposed $40 \mathrm{~km}$ Cosmic Explorer (CE) instrument [52-54] (CE2 from [95]). For EoS inference we consider only SNR above $400 \mathrm{~Hz}$ in the source frame (orange line in Fig. 1) restricting to the tidally corrected signal. Figure 2 shows the total information per redshift slice per year for $D=\{1,2\}$; at each redshift bin we add $\mathrm{SNR}^{2 D}$ from detectable binaries, representing the total information from that distance.

For the inspiral signal in the stationary phase approximation [96], the SNR is proportional to $\mathcal{M}^{5 / 6} / D_{L} \sim$ $(1+z)^{5 / 6} / D_{L}$, where $D_{L}$ is the luminosity distance and

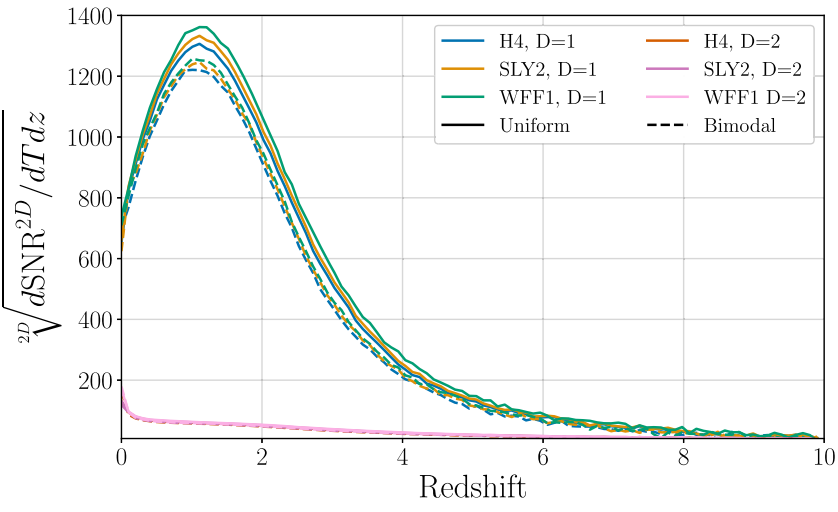

FIG. 2. Total premerger SNR distribution per year per redshift slice, as a function of redshift for two mass distributions, three EoSs, $D=\{1,2\}$, and the $40 \mathrm{~km} \mathrm{CE}$ detector. For $D=1$, the available SNR is dominated by BNSs at cosmological distances. For $D=2$, the accumulated information peaks at $z \sim 0$, suggesting a main contribution from local sources.
$\mathcal{M} \equiv\left(m_{1} m_{2}\right)^{3 / 5}\left(m_{1}+m_{2}\right)^{-1 / 5}$ is the source-frame chirp mass. As $D_{L}$ increases, the denominator increases linearly; distant signals are weaker. Simultaneously, the redshifted mass is higher than the source-frame mass resulting in a higher signal amplitude and as there are more sources at large distances the $\mathrm{SNR}^{2}$ distribution peaks at $z \sim 1$. The $\mathrm{SNR}^{4}$ distribution, on the other hand, is a monotonically decreasing function, as the $\sim 1 / D_{L}^{4}$ decline cannot be compensated by either redshifting or volume effects. For $D=1$, corresponding to measuring a single EoS scale, information is dominated by events at $z \sim 1$. For $D>1$ the distribution peaks at $z=0$ and rare loud events dominate, consistent with [79].

Besides the differing shapes of the distributions, the total accumulated SNR is also higher for $D=1$ for all EoSs and mass distributions. For a set of $N$ observations the accumulated information grows as $N^{1 /(2 D)}$, suggesting a more efficient collection of information for lower-dimensional analyses. The effect of the assumed mass distribution and EoS is smaller, though the uniform mass distribution leads to larger SNRs as it contains more massive NSs. Stiff EoSs - such as H4 - predict large NS radii for a given mass and an earlier tidal disruption relative to a softer EoS, corresponding to a small reduction in SNR.

Postmerger signal.-The postmerger signal is dominated by a single frequency component, see Fig. 1, that depends on the EoS stiffness. A subdominant structure exists [37,97], but the main subdominant modes are related to the dominant one $[32,98]$. We therefore use $D=1$ and follow a similar procedure as above, using only systems detectable by their premerger signal. For the detected signal we employ equal-mass simulations with EoS6, EoS7, and EoS4 from [50], constructed to be consistent with GW170817 [10]. Simulations were performed with a relativistic smooth particle hydrodynamics code, with conformal flatness to solve the Einstein equations [99-102].

We assume the same mass and orientation distributions as above. The postmerger signal amplitude is generally expected to increase with mass and drop steeply when the total sourceframe mass $M$ approaches the threshold for prompt $\mathrm{BH}$ formation. Reference [103] presents a phenomenological model for the postmerger signal with the amplitude proportional to observed $M$ though with some residual dependence on $q$ [103-105]. Our signals are based on numerical simulations with a certain intrinsic $M$ [50]; to estimate the observed signal from a binary with a different mass and redshift, we scale the amplitude proportionally to the redshifted $M$ [103]. We adjust the dominant frequency inversely proportionally to the system's redshifted $M$. Systems whose source-frame mass exceeds an EoS-dependent threshold result in prompt collapse, emitting a negligible signal. We quantify this threshold following [106,107] and discard systems above it from further analysis.

Figure 3 shows the total information as a function of the redshift for all EoSs and mass distributions. The available 


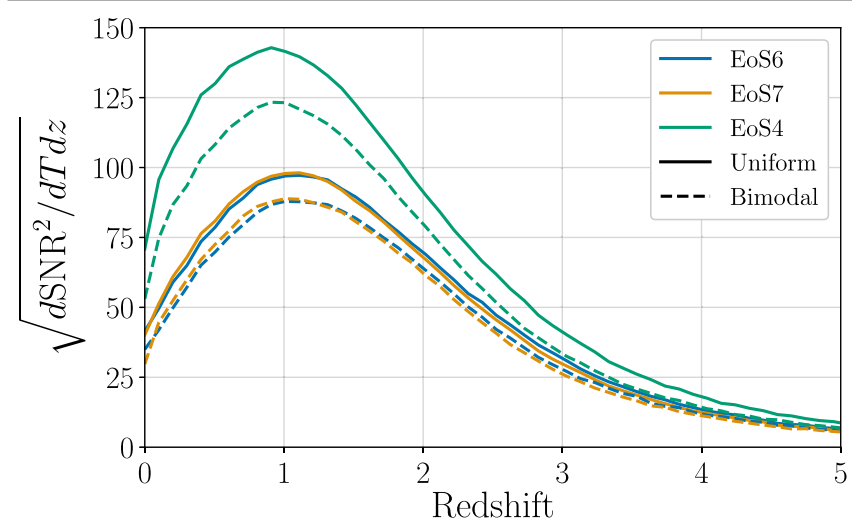

FIG. 3. Total postmerger SNR distribution per year per redshift slice as a function of redshift for two mass distributions, three EoSs and the $40 \mathrm{~km} \mathrm{CE}$ detector $(D=1)$. The distribution peaks at $z \sim 1$, suggesting a significant contribution from nonlocal sources on the total postmerger information.

postmerger SNR is dominated by nonlocal sources with a distribution peaking at $z \sim 1$. The uniform mass distribution results in an overall larger detected SNR, as it results in heavier systems and louder signals. Additionally, stiffer EoSs generally result in a higher total SNR as their postmerger frequency is lower. However, the threshold mass is also important, as a low threshold to prompt collapse means that the heavier (and potentially louder) systems emit no relevant signal. Hence the stiffest EoS in our set, EoS4, indeed reports the highest SNR, with no clear distinction between EoS6 and EoS7.

Depending on the shape of the postmerger spectrum and the steepness of the detector noise, the authors of [50] found that the lower-frequency subdominant modes might result in higher SNR than the dominant one, leading to a "reversal" in which is observed first. Combining multiple sources at different masses and redshifts could mitigate any confusion arising from a misidentification of the dominant mode from a loud source.

Effect of Detector configuration.-The conclusion that nonlocal binaries contribute significant information about finite-size effects hinges on detectors with cosmological reach for BNSs that detect signals with $z \gtrsim 1$. We repeat the above analyses with five additional detector configurations, see Fig. 1. Besides the $40 \mathrm{~km} \mathrm{CE}$ detector, we study its $20 \mathrm{~km}$ version $[52,108]$ with nominally greater sensitivity above $2 \mathrm{kHz}$ [109]. We also include the Einstein Telescope (ET) $[55,56,110]$ (in its ET-D configuration), a LIGO Voyager detector [110,111], and Advanced LIGO (aLIGO) at its design sensitivity [112,113]. Finally, we include NEMO/ OzHF [114-116], a proposed detector for dedicated highfrequency $\mathrm{GW}$ observations.

The total premerger information with each detector is shown in Fig. 4, for a single-parameter analysis assuming the bimodal mass distribution and the H4 EoS. The next generation instruments $\mathrm{CE}$ and ET detect BNS signals at cosmological distances, and nonlocal sources from $z \sim 1$

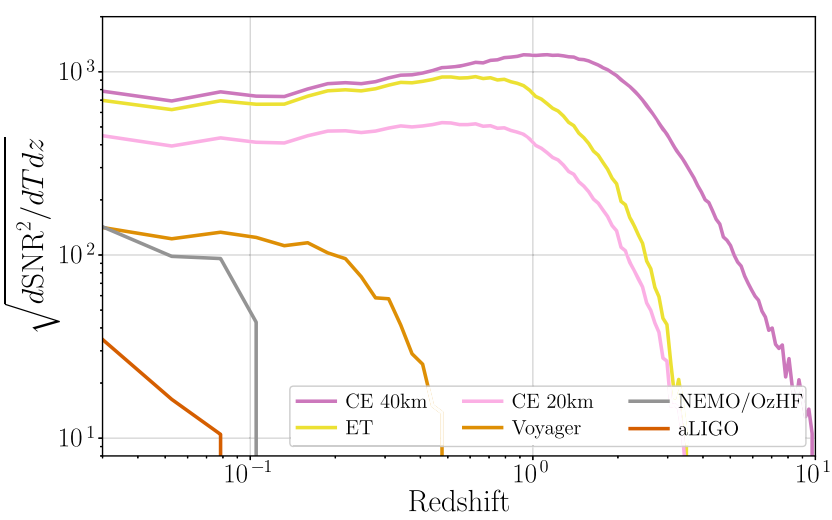

FIG. 4. Total premerger SNR distribution per year per redshift slice, as a function of redshift for the bimodal mass distribution, the H4 EoS, and for different planned or proposed detectors. We show results for $D=1$.

dominate the accumulated information. For detectors that cannot detect the myriad of distant binaries, though, the available SNR is dominated by rare loud signals.

The area under the square of each curve in Fig. 4 corresponds to the total information, or total $\mathrm{SNR}^{2}$, collected during one year of observation. Comparing the relative information gained between different detectors we can gauge their respective contributions towards characterization of the NS EoS. Table I presents the inverse relative information gained from each detector compared to CE $40 \mathrm{~km}$ for premerger $(D=\{1,2\})$ and postmerger signals. The ratios amount to years of observation with each detector to match one year of CE $40 \mathrm{~km}$ observation, or equivalently the number of detectors required to match a single CE $40 \mathrm{~km}$ instrument.

Third generation detectors offer at least an order of magnitude more information about both premerger and postmerger signals compared to noncosmological BNS detectors. The increased high-frequency sensitivity for the $20 \mathrm{~km} \mathrm{CE}$ configuration results in approximately similar performance compared to its $40 \mathrm{~km}$ counterpart for postmerger, but not for premerger, signals. "2.5" G detectors such as NEMO/OzHF and Voyager offer a clear improvement over aLIGO, and could potentially lead to a

TABLE I. Inverse relative information $\sim \mathrm{SNR}^{2 D}$ gained from each detector compared to CE $40 \mathrm{~km}$. We use results with the bimodal mass distribution and EoSs $\mathrm{H} 4$ and EoS7 for the premerger and postmerger signals respectively.

\begin{tabular}{lrrr}
\hline \hline Detector & $\begin{array}{c}\text { Premerger, } \\
D=1\end{array}$ & $\begin{array}{c}\text { Premerger, } \\
D=2\end{array}$ & $\begin{array}{r}\text { Postmerger, } \\
D=1\end{array}$ \\
\hline CE 40 km & 1 & 1 & 1 \\
ET & 3 & 2 & 9 \\
CE 20 km & 10 & 12 & 3 \\
Voyager & 830 & 1400 & 1200 \\
NEMO/OzHF & 2700 & 1100 & 450 \\
aLIGO & 64000 & 170000 & 42000 \\
\hline \hline
\end{tabular}


postmerger detection (total SNR $\gtrsim 5$ for either NEMO/ OzHF or Voyager) and stringent premerger EoS constraints [total SNR $\gtrsim 33(60)$ with $D=1$ and $\gtrsim 16(15)$ with $D=2$ for NEMO/OzHF (Voyager)]. The details of a detector's sensitivity determine whether it is more sensitive to post- or premerger signals, which can be combined to boost EoS constraints under certain assumptions. However, given the complementarity of the two signals in probing different density and temperature regimes, we choose to present them separately.

Discussion.-Over the next decade the sensitivity of GW detectors will increase, allowing for detection of a large population of cosmological BNSs. These observations are aided by the redshift-dependent merger rate as well as cosmological redshifting of the observed signals. With a conservative detection threshold of SNR $>8$ in one $40 \mathrm{~km}$ $\mathrm{CE}$ detector, we find $\sim 1 / 3$ of all BNSs in the Universe are individually detectable. In terms of SNR, they correspond to about $60 \%$ of the total available BNS SNR, as the undetected signals are subdominant following the $N^{1 / 2}$ SNR scaling. The detection procedure deployed for real GW observations is more sophisticated [117,118], including a global network of GW detectors, and potentially able to characterize both the individually resolvable signals and a stochastic background $[57,58,119,120]$, further increasing the available information.

Measurement of a single scale of the NS EoS is dominated by information from the population of BNSs at $z \sim 1$ for third-generation GW detectors. Current facilities are inherently insensitive to this distant source population and constraints are dominated by nearby sources. This suggests EoS constraints benefitting from both high and low frequency sensitivity: the high frequencies offer information about finite-size effects, the low frequencies contribute towards the detection of the distant BNS population. Extraction of more than one EoS parameter, e.g., to identify a potential phase transition [86,121], is also primarily achieved by BNSs at high individual SNR in the local Universe. The cosmological reach of third-generation detectors and the large recovered SNR for the tidally corrected signal are essential for simultaneous EoS and cosmological inference even beyond the Hubble parameter [122-124].

The large total SNR available to next-generation detectors raises the bar for mitigating systematic biases. Whether the SNR comes from a single loud source $[87,125,126]$ or a cumulative result from many weak BNSs [126], accurate models describing individual BNS waveforms are essential for unbiased estimates of the system parameters. The postmerger signal is amenable to morphology-independent studies with relaxed accuracy requirements [49]. In parallel, interpreting tidal and postmerger measurements hinges on EoS representations or universal relations. Ongoing work on improved waveform models, numerical simulations, and understanding of the EoS is essential for achieving the full potential of next-generation $\mathrm{GW}$ detectors.
The authors would like to thank Tom Callister and Georgios Lioutas for useful discussions. We also thank Daniel Brown for providing the NEMO/OzHF sensitivity curve, and Evan Hall for providing the CE $20 \mathrm{~km}$ sensitivity curve. C.-J. H. acknowledge support of the National Science Foundation, and the LIGO Laboratory. A. B. acknowledges support by the European Research Council (ERC) under the European Union's Horizon 2020 research and innovation program under Grant Agreement No. 759253. A. B. and by Deutsche Forschungsgemeinschaft (DFG, German Research Foundation), Project-ID 279384907, SFB 1245, and Project-ID 138713538, SFB 881 ("The Milky Way System", subproject A10). J. A. C. acknowledge support of the National Science Foundation Grants No. PHY-1700765, No. OAC-1841475, and No. PHY-1809572. LIGO was constructed by the California Institute of Technology and Massachusetts Institute of Technology with funding from the National Science Foundation and operates under cooperative agreement PHY-1764464. The Flatiron Institute is supported by the Simons Foundation. The authors are grateful for computational resources provided by the LIGO Laboratory and supported by National Science Foundation Grants No. PHY-0757058 and No. PHY-0823459. This analysis was made possible by the LALSUITE [127], ASTROPY [128,129], NUMPY [130], SCIPY [131], and MATPLOTLIB [132] software packages. This article carries LIGO Document Number LIGO-P2000143.

[1] J. A. Faber and F. A. Rasio, Living Rev. Relativity 15, 8 (2012).

[2] L. Baiotti and L. Rezzolla, Rep. Prog. Phys. 80, 096901 (2017).

[3] J. M. Lattimer and B. F. Schutz, Astrophys. J. 629, 979 (2005).

[4] J. Antoniadis, P. C. Freire, N. Wex, T. M. Tauris, R. S. Lynch et al., Science 340, 1233232 (2013).

[5] M. C. Miller and F. K. Lamb, Eur. Phys. J. A 52, 63 (2016).

[6] F. Özel and P. Freire, Annu. Rev, Astron. Astrophys. 54, 401 (2016).

[7] H. T. Cromartie et al., Nat. Astron. 4, 72 (2019).

[8] B. P. Abbott et al. (LIGO Scientific and Virgo Collaborations), Phys. Rev. Lett. 119, 161101 (2017).

[9] B. P. Abbott et al. (LIGO Scientific and Virgo Collaborations), Phys. Rev. X 9, 011001 (2019).

[10] B. P. Abbott et al. (LIGO Scientific and Virgo Collaborations), Phys. Rev. Lett. 121, 161101 (2018).

[11] B. P. Abbott et al. (LIGO Scientific and Virgo Collaborations), Astrophys. J. Lett. 892, L3 (2020).

[12] B. Margalit and B. D. Metzger, Astrophys. J. 850, L19 (2017).

[13] A. Bauswein, O. Just, H.-T. Janka, and N. Stergioulas, Astrophys. J. 850, L34 (2017).

[14] M. W. Coughlin, T. Dietrich, B. Margalit, and B. D. Metzger, Mon. Not. R. Astron. Soc. 489, L91 (2019).

[15] G. Raaijmakers et al., Astrophys. J. Lett. 893, L21 (2020). 
[16] P. Landry, R. Essick, and K. Chatziioannou, Phys. Rev. D 101, 123007 (2020).

[17] G. Pratten, P. Schmidt, and T. Hinderer, Nat. Commun. 11, 2553 (2020).

[18] S. Ma, H. Yu, and Y. Chen, Phys. Rev. D 101, 123020 (2020).

[19] N. N. Weinberg, Astrophys. J. 819, 109 (2016).

[20] B. P. Abbott et al. (LIGO Scientific and Virgo Collaborations), Phys. Rev. Lett. 122, 061104 (2019).

[21] É. É. Flanagan and T. Hinderer, Phys. Rev. D 77, 021502 (R) (2008)

[22] X. Zhuge, J. M. Centrella, and S. L. W. McMillan, Phys. Rev. D 50, 6247 (1994).

[23] M. Shibata, K. Taniguchi, and K. Uryu, Phys. Rev. D 71, 084021 (2005).

[24] M. Shibata and K. Taniguchi, Phys. Rev. D 73, 064027 (2006).

[25] R. Oechslin and H.-T. Janka, Phys. Rev. Lett. 99, 121102 (2007).

[26] K. Hotokezaka, K. Kyutoku, H. Okawa, M. Shibata, and K. Kiuchi, Phys. Rev. D 83, 124008 (2011).

[27] A. Bauswein and H.-T. Janka, Phys. Rev. Lett. 108, 011101 (2012).

[28] A. Bauswein, H. T. Janka, K. Hebeler, and A. Schwenk, Phys. Rev. D 86, 063001 (2012).

[29] K. Hotokezaka, K. Kiuchi, K. Kyutoku, H. Okawa, Y.-i. Sekiguchi, M. Shibata, and K. Taniguchi, Phys. Rev. D 87, 024001 (2013).

[30] K. Takami, L. Rezzolla, and L. Baiotti, Phys. Rev. Lett. 113, 091104 (2014).

[31] S. Bernuzzi, T. Dietrich, and A. Nagar, Phys. Rev. Lett. 115, 091101 (2015).

[32] A. Bauswein and N. Stergioulas, Phys. Rev. D 91, 124056 (2015).

[33] F. Foucart, R. Haas, M. D. Duez, E. O'Connor, C. D. Ott, L. Roberts, L. E. Kidder, J. Lippuner, H. P. Pfeiffer, and M. A. Scheel, Phys. Rev. D 93, 044019 (2016).

[34] L. Lehner, S. L. Liebling, C. Palenzuela, O. L. Caballero, E. O'Connor, M. Anderson, and D. Neilsen, Classical Quantum Gravity 33, 184002 (2016).

[35] W. E. East, V. Paschalidis, and F. Pretorius, Classical Quantum Gravity 33, 244004 (2016).

[36] T. Dietrich, M. Ujevic, W. Tichy, S. Bernuzzi, and B. Brügmann, Phys. Rev. D 95, 024029 (2017).

[37] N. Stergioulas, A. Bauswein, K. Zagkouris, and H.-T. Janka, Mon. Not. R. Astron. Soc. 418, 427 (2011).

[38] A. Bauswein, N. Stergioulas, and H.-T. Janka, Eur. Phys. J. A 52, 56 (2016).

[39] N. Andersson and K. D. Kokkotas, Mon. Not. R. Astron. Soc. 299, 1059 (1998).

[40] S. De, D. Finstad, J. M. Lattimer, D. A. Brown, E. Berger, and C. M. Biwer, Phys. Rev. Lett. 121, 091102 (2018); 121, 259902(E) (2018).

[41] L. Dai, T. Venumadhav, and B. Zackay.arXiv:1806.08793.

[42] B. P. Abbott et al. (LIGO Scientific and Virgo Collaborations), Astrophys. J. Lett. 851, L16 (2017).

[43] B. P. Abbott et al. (LIGO Scientific and Virgo Collaborations), Living Rev. Relativity 19, 1 (2016).

[44] R. Lynch, S. Vitale, L. Barsotti, S. Dwyer, and M. Evans, Phys. Rev. D 91, 044032 (2015).
[45] M. Tse et al., Phys. Rev. Lett. 123, 231107 (2019).

[46] L. McCuller, C. Whittle, D. Ganapathy, K. Komori, M. Tse et al., Phys. Rev. Lett. 124, 171102 (2020).

[47] H. Yang, V. Paschalidis, K. Yagi, L. Lehner, F. Pretorius, and N. Yunes, Phys. Rev. D 97, 024049 (2018).

[48] S. Bose, K. Chakravarti, L. Rezzolla, B. S. Sathyaprakash, and K. Takami, Phys. Rev. Lett. 120, 031102 (2018).

[49] K. Chatziioannou, J. A. Clark, A. Bauswein, M. Millhouse, T. B. Littenberg, and N. Cornish, Phys. Rev. D 96, 124035 (2017).

[50] A. Torres-Rivas, K. Chatziioannou, A. Bauswein, and J. A. Clark, Phys. Rev. D 99, 044014 (2019).

[51] D. Martynov, H. Miao, H. Yang, F. H. Vivanco, E. Thrane et al., Phys. Rev. D 99, 102004 (2019).

[52] B. P. Abbott et al. (LIGO Scientific Collaboration), Classical Quantum Gravity 34, 044001 (2017).

[53] D. Reitze et al., Bull. Am. Astron. Soc. 51, 141 (2019), https://inspirehep.net/literature/1724707.

[54] D. Reitze et al., Bull. Am. Astron. Soc. 51, 035 (2019), https://inspirehep.net/literature/1743201.

[55] M. Punturo et al., Classical Quantum Gravity 27, 194002 (2010).

[56] M. Maggiore et al., J. Cosmol. Astropart. Phys. 03 (2020) 050 .

[57] S. Sachdev, T. Regimbau, and B. S. Sathyaprakash, Phys. Rev. D 102, 024051 (2020).

[58] B. P. Abbott et al. (LIGO Scientific and Virgo Collaborations), Phys. Rev. Lett. 120, 091101 (2018).

[59] B. P. Abbott et al. (LIGO Scientific and Virgo Collaborations), Phys. Rev. X 9, 031040 (2019).

[60] E. D. Hall and M. Evans, Classical Quantum Gravity 36, 225002 (2019).

[61] C. J. Moore, R. H. Cole, and C. P. L. Berry, Classical Quantum Gravity 32, 015014 (2015).

[62] B. D. Lackey, M. Nayyar, and B. J. Owen, Phys. Rev. D 73, 024021 (2006).

[63] P. Madau and M. Dickinson, Annu. Rev. Astron. Astrophys. 52, 415 (2014).

[64] M. Favata, Phys. Rev. Lett. 112, 101101 (2014).

[65] L. Wade, J. D. E. Creighton, E. Ochsner, B. D. Lackey, B. F. Farr, T. B. Littenberg, and V. Raymond, Phys. Rev. D 89, 103012 (2014).

[66] M. Vallisneri, Phys. Rev. D 77, 042001 (2008).

[67] B. Bécsy and N. J. Cornish, Classical Quantum Gravity 37, 135011 (2020).

[68] W. Del Pozzo, T. G. F. Li, M. Agathos, C. Van Den Broeck, and S. Vitale, Phys. Rev. Lett. 111, 071101 (2013).

[69] K. Yagi and N. Yunes, Classical Quantum Gravity 33, 13LT01 (2016).

[70] K. Chatziioannou, C.-J. Haster, and A. Zimmerman, Phys. Rev. D 97, 104036 (2018).

[71] C. Raithel, F. Özel, and D. Psaltis, Astrophys. J. 857, L23 (2018).

[72] B. Kumar and P. Landry, Phys. Rev. D 99, 123026 (2019).

[73] M. Oertel, M. Hempel, T. Klähn, and S. Typel, Rev. Mod. Phys. 89, 015007 (2017).

[74] G. Montaña, L. Tolós, M. Hanauske, and L. Rezzolla, Phys. Rev. D 99, 103009 (2019).

[75] R. Essick, P. Landry, and D. E. Holz, Phys. Rev. D 101, 063007 (2020). 
[76] H.-Y. Chen, P. M. Chesler, and A. Loeb, Astrophys. J. 893, L4 (2020).

[77] K. Chatziioannou and S. Han, Phys. Rev. D 101, 044019 (2020).

[78] J. S. Read, B. D. Lackey, B. J. Owen, and J. L. Friedman, Phys. Rev. D 79, 124032 (2009).

[79] B. D. Lackey and L. Wade, Phys. Rev. D 91, 043002 (2015).

[80] L. Lindblom, Phys. Rev. D 97, 123019 (2018).

[81] M. F. Carney, L. E. Wade, and B. S. Irwin, Phys. Rev. D 98, 063004 (2018).

[82] C. A. Raithel, F. Özel, and D. Psaltis, Astrophys. J. 831, 44 (2016).

[83] I. Tews, J. Carlson, S. Gandolfi, and S. Reddy, Astrophys. J. 860, 149 (2018).

[84] S. K. Greif, G. Raaijmakers, K. Hebeler, A. Schwenk, and A. L. Watts, Mon. Not. R. Astron. Soc. 485, 5363 (2019).

[85] M. G. Alford and S. Han, Eur. Phys. J. A 52, 62 (2016).

[86] S. Han and A. W. Steiner, Phys. Rev. D 99, 083014 (2019).

[87] R. Dudi, F. Pannarale, T. Dietrich, M. Hannam, S. Bernuzzi, F. Ohme, and B. Brügmann, Phys. Rev. D 98, 084061 (2018).

[88] R. B. Wiringa, V. Fiks, and A. Fabrocini, Phys. Rev. C 38, 1010 (1988).

[89] F. Gulminelli and A. R. Raduta, Phys. Rev. C 92, 055803 (2015).

[90] P. Danielewicz and J. Lee, Nucl. Phys. A818, 36 (2009).

[91] B. P. Abbott et al. (LIGO Scientific and Virgo Collaboration), Classical Quantum Gravity 37, 045006 (2020).

[92] J. Alsing, H. O. Silva, and E. Berti, Mon. Not. R. Astron. Soc. 478, 1377 (2018).

[93] M. Dominik, K. Belczynski, C. Fryer, D. Holz, E. Berti, T. Bulik, I. Mandel, and R. O'Shaughnessy, Astrophys. J. 759, 52 (2012).

[94] T. Dietrich, S. Khan, R. Dudi, S. J. Kapadia, P. Kumar et al., Phys. Rev. D 99, 024029 (2019).

[95] Cosmic Explorer Project, Cosmic explorer (2019), https:// cosmicexplorer.org/researchers.html.

[96] S. Droz, D. J. Knapp, E. Poisson, and B. J. Owen, Phys. Rev. D 59, 124016 (1999).

[97] K. Takami, L. Rezzolla, and L. Baiotti, Phys. Rev. Lett. 113, 091104 (2014).

[98] J. A. Clark, A. Bauswein, N. Stergioulas, and D. Shoemaker, Classical Quantum Gravity 33, 085003 (2016).

[99] J. R. Wilson, G. J. Mathews, and P. Marronetti, Phys. Rev. D 54, 1317 (1996).

[100] R. Oechslin, S. Rosswog, and F. K. Thielemann, Phys. Rev. D 65, 103005 (2002).

[101] R. Oechslin, H.-T. Janka, and A. Marek, Astron. Astrophys. 467, 395 (2007).

[102] A. Bauswein, H.-T. Janka, and R. Oechslin, Phys. Rev. D 82, 084043 (2010).

[103] K. W. Tsang, T. Dietrich, and C. Van Den Broeck, Phys. Rev. D 100, 044047 (2019).

[104] M. Breschi, S. Bernuzzi, F. Zappa, M. Agathos, A. Perego, D. Radice, and A. Nagar, Phys. Rev. D 100, 104029 (2019).
[105] S. Bernuzzi et al., Mon. Not. R. Astron. Soc. 497, 1488 (2020).

[106] A. Bauswein, T. W. Baumgarte, and H. T. Janka, Phys. Rev. Lett. 111, 131101 (2013).

[107] A. Bauswein, S. Blacker, V. Vijayan, N. Stergioulas, K. Chatziioannou, J. A. Clark, Niels-Uwe F. Bastian, D. B. Blaschke, M. Cierniak, and T. Fischer, Phys. Rev. Lett. 125, 141103 (2020).

[108] E. D. Hall (private communication) (2020).

[109] Both Cosmic Explorer configurations explored here assume a $1.4 \%$ transmissivity of the input test mass, and a $2 \%$ transmissivity of the signal-recycling mirror.

[110] M. Evans, R. Sturani, S. Vitale, and E. Hall, Unofficial sensitivity curves (ASD) for aLIGO, Kagra, Virgo, Voyager, Cosmic Explorer and ET, Tech. Rep. LIGO-T1500293 (2018), https://dcc.ligo.org/LIGO-T1500293/public.

[111] R. Adhikari et al. (LIGO Scientific Collaboration), Classical Quantum Gravity 37, 165003 (2020).

[112] J. Aasi et al. (LIGO Scientific Collaboration), Classical Quantum Gravity 32, 074001 (2015).

[113] L. Barsotti, P. Fritschel, M. Evans, and S. Gras, Updated Advanced LIGO sensitivity design curve, Tech. Rep. LIGO-T1800044 (2018), https://dcc.ligo.org/LIGOT1800044/public.

[114] M. Bailes et al., arXiv:1912.06305.

[115] D. D. Brown (private communication) (2020), OzGrav, LIGO DCC-T2000062.

[116] K. Ackley et al., Publ. Astron. Soc., 37 (2020).

[117] K. Cannon et al., Astrophys. J. 748, 136 (2012).

[118] D. Meacher, K. Cannon, C. Hanna, T. Regimbau, and B. S. Sathyaprakash, Phys. Rev. D 93, 024018 (2016).

[119] X.-J. Zhu, E. J. Howell, D. G. Blair, and Z.-H. Zhu, Mon. Not. R. Astron. Soc. 431, 882 (2013).

[120] F. Hernandez Vivanco, R. Smith, E. Thrane, and P. D. Lasky, Phys. Rev. D 100, 043023 (2019).

[121] A. Bauswein, Niels-Uwe F. Bastian, D. B. Blaschke, K. Chatziioannou, J. A. Clark, T. Fischer, and M. Oertel, Phys. Rev. Lett. 122, 061102 (2019).

[122] C. Messenger and J. Read, Phys. Rev. Lett. 108, 091101 (2012).

[123] C. Messenger, K. Takami, S. Gossan, L. Rezzolla, and B. S. Sathyaprakash, Phys. Rev. X 4, 041004 (2014).

[124] W. Del Pozzo, T. G. F. Li, and C. Messenger, Phys. Rev. D 95, 043502 (2017).

[125] A. Samajdar and T. Dietrich, Phys. Rev. D 98, 124030 (2018).

[126] M. Pürrer and C.-J. Haster, Phys. Rev. Research 2, 023151 (2020).

[127] LIGO Scientific Collaboration, LIGO Algorithm LibraryLALSuite, free software (GPL) (2018), https://doi.org/10 .7935/GT1W-FZ16.

[128] T. P. Robitaille et al. (AstropyCollaboration), Astron. Astrophys. 558, A33 (2013).

[129] A. Price-Whelan et al., Astron. J. 156, 123 (2018).

[130] C. R. Harris et al., Nature (London) 585, 357 (2020).

[131] P. Virtanen et al., Nat. Methods 17, 261 (2020).

[132] J. D. Hunter, Comput. Sci. Eng. 9, 90 (2007). 